East African Medical Journal Vol. 79 No. 5 May 2002

CELLULAR AND MOLECULAR INTERACTIONS IN HIV INFECTIONS: A REVIEW

A. G. Tumbo-Oeri, BSc. (Hons), PhD, OGW, Associate Professor and C. A. Omwandho, BSc (Hons), MSc, PhD, Lecturer, Department of Biochemistry, College of Biological and Physical Sciences. University of Nairobi, P.O. Box 30197, Nairobi, Kenya.

Request for reprints to: Prof. A.G. Tumbo-Oeri, Principal's Office, College of Biological and Physical Sciences, University of Nairobi, P.O. Box 30197, Nairobi, Kenya.

\title{
CELLULAR AND MOLECULAR INTERACTIONS IN HIV INFECTIONS: A REVIEW
}

\section{A. G. TUMBO-OERI and C. A. OMWANDHO}

\begin{abstract}
Objective: To review the cellular and molecular interactions between HIV and the host immune system that lead to full-blown AIDS.

Data Sources: Published reports on HIV/host interaction during a fifteen year period beginning from 1987.

Study selection: Only those studies involving humans and non-human primates were selected. The studies included original articles and state-of-the-art reviews covering in vivo and in vitro findings.

Data extraction and synthesis: This article presents a critical review of the cellular and molecular mechanisms of HIV infection and their relationship to the onset of AIDS.

Conclusion: HIV has elaborated diverse and somewhat complicated mechanisms for the subversion and evasion of the host immune defence strategies. These include escape through mutation, prolonged latency of the infection, masking of the viral envelope proteins, downregulation of MHC-I and up-regulation of the Fas-ligand on infected cell surfaces. This review enhances our understanding of HIV/AIDS disease and presents a basis on which management strategies could be developed.
\end{abstract}

\section{INTRODUCTION}

The human immunodeficiency virus (HIV- 1) infects, functionally impairs and depletes a sub-population of thymus-derived T-lymphocytes that express the cellsurface molecule CD4t. The latter perform critical recognition and induction functions in the immune response to foreign stimuli. HIV-1 infection results in gradual $\mathrm{CD} 4+\mathrm{T}$ cell depletion, progressive immune unresponsiveness with effective paralysis in virtually all arms of the immune system and increasing susceptibility to opportunistic infections and malignancies. The clinical spectrum of HIV infection ranges from asymptomatic infection to severe immune deficiency with the infections/ malignant complications that are characteristic of the acquired immunodeficiency syndrome (AIDS).

This spectrum of clinical severity is reflected in a parallel severity in CD4+ T cell depletion. Thus HIV-1 immunodeficiency is viewed from the immunological perspective as a graded severity of $\mathrm{CD} 4+\mathrm{T}$ cell depletion, distributed on a continuum of time, influenced by poorly understood factors and associated with a spectrum of increasing clinical severity that reflects the severity of $\mathrm{CD} 4+\mathrm{T}$ cell depletion.

Individuals infected with HIV mount and sustain a vigorous immune response to HIV- 1 . Cells infected by HIV-1 are normally recognised and eliminated by the immune system. One key to this phenomenon is the surface presentation of viral peptides by proteins of the major histocompatibility complex (MHC) allowing their detection and killing by cytotoxic T-Iymphocytes (CTLs). This process initially functions well but achieves only temporary success(1). A strong cytotoxic T cell response can be detected early after infection with upto $1-5 \%$ of the pool CD8+ T cells specific for the virus(2). While this is apparently responsible for the abrupt drop in plasma viremia that occurs within a few weeks of infection, it ultimately fails to contain the infection. Behind this failure is the tale of a hunter that becomes the hunted and of a prey that masters the art of delusion. Progressively, HIV destroys the helper $T$ lymphocyte pool thereby precluding both the efficient production of anti- HIV antibodies by B cells and the proper functioning of CTLs(3).

In parallel, the virus exploits a greater diversity of strategies to escape both the humoral and cellular arms of the immune response. These evasive strategies are the subject of this communication. They include mutational escape, latency, masking of antibody binding sites on the viral envelope, down-modulation of the class I histocompatibility complex (MHC-I) and upregulation of the Fas ligand on the surface of infected cells. Examining the mechanisms of these phenomena facilitates our understanding of how HIV wins its war against the immune system and suggests avenues for combating the virus through chemotherapy and vaccine development.

Mutational escape: The human and simian immunodeficiency viruses (HIV and SIV) are members of the lentiviridae subgroup of retroviruses that cause progressive failure of the host immunological functions culminating in the clinical collapse called AIDS. In the absence of antiviral therapy, this course is inexorable in spite of an initially vigorous immune response. Two fundamental characteristics of the biology of primate lentiviruses explain this apparent paradox. One, HIV and SIV infect CD4+ targets such as helper $\mathrm{T}$ Iymphocytes and macrophages, cells that normally play an essential role in the emergence and maintenance of an 
effective antiviral response. Two, these viruses have evolved a number of strategies to evade control by the immune system. Their antigenic repertoire is capable of a formidable pleiomorphism owing to the lack of fidelity of viral reverse transcriptase (RT) and to a remarkable degree of tolerance for sequence variability particularly in the viral envelope. On average, each time the $10 \mathrm{~Kb}$ HIV genome is replicated, one nucleotide substitution is introduced(4). Millions of new viruses, reflecting an almost complete turnover of the circulating pool are generated every day $(5,6)$. The selective pressure exerted on this viral population by the immune response quickly leads to an antigenic drift in many viral proteins, on epitopes recognised either by neutralising antibodies or by cytotoxic T Iymphocytes(7). Monkeys infected with an SIV clone of defined sequence accumulated nucleotidechanges in Env-at a rate of $1 \%$ peryear correlating with the emergence of variants that could escape the effect of initially neutralizing antibodies $(8)$. Similarly, a progressive variation within dominant epitopes of the envelope (Env) and Nef viral proteins, allowing the virus to progressively evade the recognition by specific CTLs was observed in SIVinfected monkeys (9). Furthermore, the mutation of these viral epitopes significantly decreased the efficiency of CTL - mediated killing of infected cells presenting such peptides(1). During the course of an infection, the immunoselection of escape variants correlated with the emergence of viral populations with an increased fitness or replication in the infected animal(10). CTL-escape variants are alsodocumented in HIV - infected individuals for instance in the immediate aftermath of the primary phase of the infection in a patient with strong B44 - restricted response to an immunodominant Env epitope(11).

\section{ESCAPE FROM THE CLAWS OF NEUTRALIZING ANTIBODIES}

In in vitro assays, high levels of antibodies against the viral envelope can be detected in the plasma of both SIV and HIV - infected subjects. Nonetheless, neutralising antibodies seem to be rare particularly when dealing with primary viral isolates. Three possible explanations can be advanced for this paradox. First, many antibodies are directed against the monomeric, soluble form of the envelope. Such antibodies react only poorly if at all with the envelope oligomer that is found on the surface of various infected cells (12). Second, the viral envelope is heavily glycosylated. In fact, close to $50 \%$ of the mass of gp 120 , the surface moiety of the HIV - 1 envelope is carbohydrate. This modification results in masking of critical epitopes(13). Third, antibodies with some of the strongest neutralising potential target regions of the envelope that are transiently exposed at the time of viral entry, when the glycoprotein is ready to mediate the fusion between viral and cellular membranes $(14,15)$. These observations have guided efforts aiming at generating better neutralising antibodies. Some have relied on the use of cells expressing a form of Env locked in a fusion competent state as immunogens(16). Although these antibodies could inhibit a broad number of primary HIV1 isolates, their specificity for given regions of the viral envelope remains to be demonstrated.

\section{SHELTER FROM THE CELLULAR IMMUNE RESPONSE: LATENCY}

HIV can apparently hide from the attacks of cytotoxic $T$ Iymphocytes in at least two sites: the glial cell of the central nervous system (CNS), an organ where cellmediated responses are typically non-existent or dimmed and the resting $\mathrm{T}$ lymphocyte. In the latter case, the viral genome is integrated in the chromosome while remaining unexpressed or lowly transcribed in the absence of activation. Studies of patients treated with highly active anti-retroviral therapy (HAART) have shed some light on this latent reservoir. Many of these individuals exhibit exceedingly low levels of plasma viremia, often below the limits of detection of current assays. However, viruses can be readily recovered from their peripheral blood mononuclear cells (PBMCs) following $T$ cell receptor (TCR) stimulation(17). A closer examination of unstimulated PBMCs from patients on HAART revealed the presence of episomal HIV-I DNA intermediates, a strong indicator of ongoing viral replication(18). This finding has two implications: One, it shows that in some compartments, HIV can defy very potent drug treatments. Two, it demonstrates that some virus-expressing cells are not efficiently recognised by the immune system in spite of residing in theoretically accessible locations.

\section{MHC-I DOWN MODULATION}

It is now established that HIV can escape the attacks of CTLs by down-regulating the expression of MHC-I on the surface of infected cells(19). This strategy is not unprecedented being also exploited by several other viruses including the Epstein - Barr virus (EBV), the cytomegalovirus (CMV), the herpes simplex virus (HSV) and adenovirus $(20)$. In the case of HIV, the main culprit is a viral factor called Nef although another protein called $V$ pu may also play a minor role(21). Nef is a short cytoplasmic protein that associates with membranes through $\mathrm{N}$-terminal myristoylation and exerts several additional effects on the infected cell. For instance, it is responsible for downregulating the surface expression of $\mathrm{CD} 4+$ the main HIV receptor(22). Nef down-modulates $\mathrm{MHCI}$ in a variety of cell types including primary $\mathrm{T}$ Iymphocytes.

MHC-I is composed of a highly polymorphic, membrane -anchored heavy chain non-covalently associated with $\beta 2$ microglobulin $(\beta 2 \mathrm{~m})$. The assembly of the heavy chain with $\beta 2 \mathrm{~m}$ occurs in the endoplasmic reticulum (ER) or in the cis - Golgi apparatus where antigenic peptides are loaded(23). This loading is necessary for the transport of the complex to the cell surface. In the presence of Nef, MHC-I is normally synthesized and transported through the ER and cis-Golgi and can reach the cell surface. However, it is then diverted towards the endosomal pathway to accumulate in the transGolgi network (TGN) before undergoing degradation(24). 
Determinants necessary for $\mathrm{Nef}$ responsiveness are contained in the cytoplasmic domain of MHC-I and are centered around a critical tyrosine residue found in HLA-A and $B$ but not in HLA-C(25). Correspondingly, HLA-C is unaffected by Nef(26). This may be of physiological importance because HLA-Cmolecules are dominant inhibitory ligands that protect cells against lysis by natural killer (NK) lymphocytes which normally destroy MHC-I negative cells(27). Mutagenesis studies have pointed to the importance of specific determinants in the ability of HIV- $1 \mathrm{Nef}$ to regulate MHC-I. These include the Nef myristoylation signal, essential for its membrane association, an N-terminal $\alpha$-helix, a centrally located SH3binding proline-based repeat and a highly conserved cluster of acidic residues in the $\mathrm{N}$-proximal third of the viral protein $\{\operatorname{EEEE}(65)\}(28)$. The acidic cluster (AC) of Nef is highly similar to the PACS- 1 binding, TGN retrieval motif of furin and mannose- 6 phosphate receptor (MPR). PACS-1 (phosphofurin acidic cluster sorting protein-l) is the first identified member of a new family of coat proteins. PACS1 governs the endosometo-Golgitrafficking of furin and MPR by connecting the $\mathrm{AC}$-containing cytoplasmic domain of these molecules with the adapter protein complex (AP-1) of endosomal clathrin-coated pits(CCP)(29). Correspondingly, the AC-mediated recruitment of PACS- 1 is responsible for MHC-Idown regulationand TGN targeting (24). Furthermore, a chimeric integral membrane protein harbouring Nef as its cytoplasmic domain localizes to the TGN after internalization in an AC- and PACS-I dependent manner (24). These results support a model in which Nef down regulates MHC-I by acting as a connector between the receptor cytoplasmic tail and the PACS- 1 sorting pathway (Figure 1). An actual interaction between Nef and MHC-I remains to be demonstrated.

Figure 1

Nef-induced receptor down-regulation in HIV infection

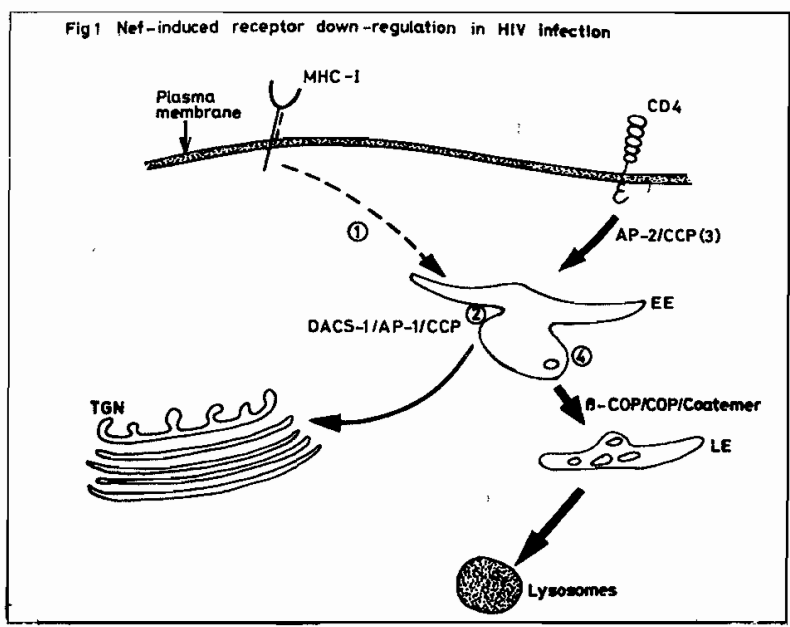

Nef accelerates the internalization of cell surface MHC through an unknown mechanism and pathway.

2. In the endosome, the viral protein connects the MHC Class I complex wilh PACS-1 an adaptor molecule that Iccruits the adapter protein complex (AP-1) thereby governing the clathrin-coated pits (CCP)-mediated transGolgi network (TGN) retrieval of MHC.

3. Net connects CD4 with AP-2 adaptor protein complex, triggering the formation of CD4specific clatrin coated vesicles that rapidly internalize the receptor

4. In the early endosome (EE) Nef bridges CD4 with the endosomal COP-I coatemer that embarks the HIV receptor for degradation in late endosome (LE)/hysosomes.
This connector model is a recurrent theme for Nef. It is the very mechanism by which the viral protein induces the down-regulation of $\mathrm{CD} 4$, the main HIV receptor. However, in this other case, both the downstream partners of Nef and the fate of its cellular target are different. In the Golgi, and the plasma membrane, Nef bridges the cytoplasmic tail of $\mathrm{CD} 4$, receptor molecules with the adaptor protein complex of clathrin coated pits, thereby, triggering the formation of $\mathrm{CD} 4$-specific endocytic vesicles. Then, in the early endosome, Nef connects CD4 receptors with a subset of COP-I coatomers that embark the receptor for degradation in Iysosomes (30). Nef-induced CD4 down regulation contributes to the fitness of viral replication, since it preserves the infectivity of HIV-I virions by preventing an interference between the receptor and particle release or envelope incorporation (31).

\section{NEF-INDUCED FAS LIGAND UPREGULATION}

The depletion of $T$ cell function that accompanies the late phase of HIV infection results partly from a high degree of apoptosis undergone not only by the CD4+ viral targets but also by CD8+ Iymphocytes(32). Much of this apoptosis is mediated by the Fas-Fas ligand (FasL) interactions (Figure 2). In HIV-infected patients, there is upregulation of both Fas and FasL as well as an increased susceptibility of CD4+ and CD8+ cells to Fasmediated killing(33). Furthermore, both SIV and HIV can upregulate the expression of FasL on the surface of infected cells(34).

Figure 2

Nef-induced Fas-ligand upregulation

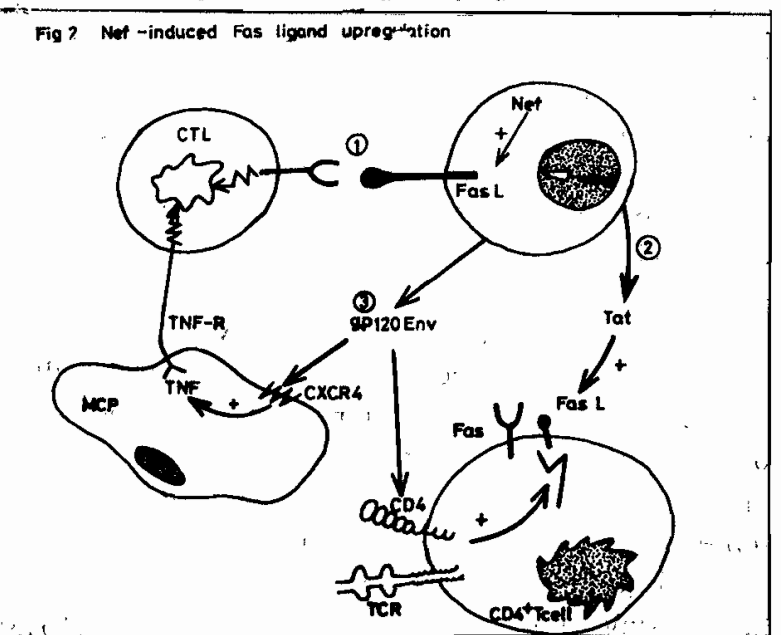

Ner upregulates the expression of Fas ligand (Fas $L$ ) on the surface of HIV nntected cells

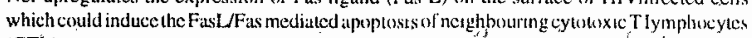
(CTL).

2. Tal produced by infected cells can sensitize neiglbourng CD4+ T. Iymphocyless apoptosis, Cross-linking of CD4 by HIV Env in the presence of the viral utansati vilter Tyll can induce Fas Lexpression and apoptosis of uninfected helper $T$ cells.

3. The interaction ol Eov with the chemokine receptor CXCR4 on the macrophage (MCP) leads to the death of bystunder $\mathrm{CD} 8+\mathrm{T}$ cells via the induction of tumour necrosis factir (TNF) and the activation of its cognatc receptor. 
The mediator of this effect is $\operatorname{Nef}(34)$ while two other viral proteins Tat and Env act on bystander cells(35). Nefinduced FasL upregulation which proceeds through an incompletely characterised activation of the T cell receptor pathway may play an important role in the progressive loss of the antiviral immune response. Other Fas-related events contribute to HIV-induced cell death of bystander lymphocytes. For example, crosslinking of CD4 by HIV Env in the presence of the viral transactivator Tat can induce FasL expression and apoptosis of uninfected $T$ cells(35). Furthermore, interaction of Env with the chemokine receptor CXCR4 on macrophages leads to the death of bystander CD8 $+\mathrm{T}$ cells via the induction of tumour necrosis factor (TNF) and activation of its cognate receptor(36).

\section{CONCLUSION}

When it was established that HIV infects CD4+ T Iymphocytes, the mechanisms by which the virus wins its war against the immune system appeared to be quite obvious. It probably reflected a progressive exhaustion of the immune response through the direct killing of the helper T cells. Although considerable progress has been made, many questions remain unanswered and what is known is not yet adequate for translation into effective preventive strategies such as vaccination. Besides HIV, all viruses known to downmodulate MHC-I encode factors that interfere with the assembly and transport of this complex along the exocytic pathway. The Nef-induced diversion of class I molecules from the cell surface is therefore unprecedented. Equally intriguing is the fact that Nef redirects MHC-I to the TGN instead of simply sending the receptor to Iysosomes like it does for CD4. This dual originality strongly suggests that the Golgi targeting of MHC-I is advantageous for HIV. It could be that TGNretriever MHC-I molecules exert a dominant negative effect on class I complexes not directly influenced by Nef. Addressing this question is likely to reveal important information on MHC-I mediated antigen presentation. It is an area that is ripe for further research.

Nef is one of the earliest HIV gene products. It is also expressed in some forms of viral latency. This may be particularly relevant in the reservoir of infected PBMCs that persists in patients treated with HAART. It could well be that Nef helps these cells to escape detection and killing by CTLs. Because this cell population represents an obstacle to the eradication of the virus, drugs aimed at blocking Nef-induced MHC-I down regulation could represent useful complements to more conventional antiviral therapies. Also, efforts aimed at inducing strongly neutralising antibodies whether based on the hypoglycosylated envelope immunogens or artificial forms of the viral surface protein that mimic some Env intermediates should be actively pursued. Cumulative evidence points to the crucial role of cell-mediated immunity (CMI) in the control of HIV. Specifically, a strong CD4-specific antiviral proliferative activity seems to correlate with the long-term non-progression of the infection(3). It is difficult to understand how such a strong response can be triggered when activated CD4 cells are attracted to a locus of viral replication and are ideal targets for the virus. It is abundantly clear that outsmarting of HIV by the immune response is a daunting undertaking which unfortunately must be won through overcoming the malice of immunological evasion.

\section{REFERENCES}

1. Schmits, J.E. et al. Control of viremia in simian immunodeficiency virus infection by CD8+ Iymphocytes. Science. 1999; 283:857-860

2. . Ogg, G S. Quantitation of HIV-I specific cytotoxic T Iymphocytes and plasma load of viral RNA. Science. 1998; 279:2103-2106

3. Rosenberg, E.S., Billingsley, J.M., Caliendo, A.M., Boswell, S.L., Sax, P.E., Kalams, S.A. and Walker, B.D. Vigorous HIV - I specific CD4+ T cell responses associated with control of viremia. Science. 1999; 278: 1447-1450.

4. Masnky, L. M. and Temin, H. M. Lower in vivo mutation rate of human immunodeficiency virus type- $]$ than that predicted from the fidelity of purified reverse transcriptase.

5. Wei X; Ghosh SK; Taylor M.E; Johnson V.A. et al. Viral dynamics in human immunodeficiency type 1 infection. Nature. 1995: 373: 117-122.

6. Ho, D., Neumann, A., Perelson, A., Chen, W., Leonard, J. and Markowitz M. Rapid turnover of plasma virions and CD4 Iymphocytes in HIV infection. Nature. 1995; 373: 123-126.

7. McMichael, A. T-cell responses and viral escape. Cell. 1998; 93:673-676.

8. Burns, D. P. and Desrosiers, R. C. Selection of genetic variants of simian immunodeficiency virus in persistently infected rhesus monkeys. J. Virol. 1991; 65: 1843-1854.

9. Burns, D.P., Collignin C. and Desrosiers, R.C. Simian immunodeficiency virus mutants resistant to serum neutralization arise during persistent infection of rhesus monkeys. J. Virol. 1993; 67:4104 - 4113 .

10. Evans, D. T. Virus specific cytotoxic $\mathrm{T}$ Iymphocyte responses select for amino acid variation in simian immunodeficiency virus Env and Nef. Nat. Med. 1999; 5:1270 - 1276.

11. Kimata, J. T., Kuller, L., Anderson, D. B., Dailey, P. and Overbaugh, I. Emerging cytopathic and antigenic Simian immunodeficiency virus variants influence AIDS progression. Nat. Med. 1999; 5: 5335 - 5341.

12. Moore, J. P., Cao, Y., Qing, L.. et al. Primary isolates of human immunodeficiency virus type $I$ are relatively resistant to neutralization by mono clonal antibodies is not predicted by studies with monometric gp 120. J.Virol. 1995; 69: 101-109.

13. Sattentan, Q. J. and Moore J. P. Human immunodeficiency virus type I neutralization is determined by epitope exposure on the jp 120 obligomer. J. Exp. Med. 1995; 182:185-196.

14. Reitter, J. N., Means, R. E. and Desrosiers, R. C. A role for carbohydrates in immune evasion in AIDS. Nat. Med. 1998; 4: 679 - 684.

15. Weissenhorn, W., Dessen, A., Harrison, S. C., Skehel J. J. and Wiley D. C. Atomic structure of the ectodomain from HIV - $\operatorname{lgp} 41$. Nature. 1997; 387: 426 - 430.

16. Kwong, P. D., Wyatt, R., Robinson, J., Sweet, R. W., Sodroski, J. and Hendrickson, W. A. Structure of an HIV - GP 120 envelope glycoprotein in complex with CD4 receptor and neutralizing human antibody. Nature. 1998; 393: 648 - 659 .

17. Finzi, D. Identification of a reservoir for HIV - I in patients on highly active antiretroviral therapy. Science. 1998; 278:1295 . 1300.

18. Ramratnam, B., Mittler, J.E., Zhang, L. et al. The delay of the latent reservoir of replication - competent HIV - 1 is inversely correlated with the extent of residual viral replication during prolonged antiretroviral therapy. Nat. Med. 2000; 6: 82 - 85 .

19. Ploegh, H L. Viral strategies of immune evasion. Science. 1998 ; 280:248 - 253.

20. Brodskij F. M., Lem L., Solache A. and Bennett E. M. Human 
pathogen subversion of antigen presentation. Immunol. Rev. 1999; 168: 199 - 217.

21. Kerkau T., Bacik I., Bennink J., Yewdell J., Hunig T., Schimpl A. and Schubert $U$. The human immunodeficiency virus type I (HIV -1) Vpu protein interferes with an early step in the biosynthesis of major histocompatibility complex (MHC) class I molecules. J. Exp. Med. 1997; 185:1295-1305.

22. Guy B., Kieny M., Riviere Y., Peuch C., Dott K., Girard M., Montagnier $L$. and Lecocq J. HIV F/3 of encodes a phosphorylated GTP-binding protein resembling an oncogene product. Nature. 1987; 330:266 - 269 .

23. Bylmakers $M \mathrm{~J}$, Ploegh $\mathrm{H} \mathrm{L}$, Putting together an MHC Class 1 molecule Curr. Opin. Immunol. 1993; 5:21-26.

24. Piquet V., Wan L., Borel C., Mangasanian A., Demaurex N., Thomas G. and Trono D. HIV -1 Nef protein binds to the cellular protein PACS - I to down regulate class I major histocompatibility complexes Nat. Cell. Biol. 2000; 2: $163-167$.

25. Parham P., Adams E. J. and Arnett K. L. The origins of HLA -ABC polymorphism. Immunol. Rev. 1995; 143:141-180.

26. Cohen, G.B., Gandhi R. T., Davis D. M., Mandelboin O., Chen B. K., Strominger J. L and Baltimore D. The selective down regulation of class I major histocompatibility complex proteins by HIV - I protects HIV - infected cells from N K cells. Immunity, 1999; 10: $661-671$.

27. Brutkiewicz R. R, and Welsh R. M. Major histocompatibility complex class I antigens and the control of viral infections by natural killer cells. J Virol. 1995; 69: 3967 - 3971.

28. Geenberg M. E., Iatrate A. J. and Skowronski J. The SH3 domain binding surface and an acidic motif in HIV - 1 . Nef regulates trafficking of class I MHC complexes. EMBO. $J, 1998 ; 17: 2777$ - 2789.

29. Molloy S. S., Andersar E. D., Jean F. and Thomas, G. Bicycling the furin pathway: from $T$ localization to pathogen activation and embryogenesis. Trends Cell Biol. 1999; 9:28 - 35 .

30. Schwartz O., Dantry V. A., Goud B., Marechal V., Subtil A., Heard J. M. and Danos O. Human immunodeficiency virus type I Nef induces accumulation of CD4 in early endosons. J. Virol 1995; 69: $528-533$

31. Lama J., Mangasarian A. and Trono D. Cell surface expression of CD4 reduces HIV-I infectivity by blocking Env, incorporation in a Nef - and vpu inhibitable manner. Curr Biol. 1999; 9:622 - 631.

32. Muro - Cacho, C. A., Pantaleo, G. and Fauci, A. S. Analysis of apoptosis in Iymph nodes of HIV-infected persons. Internity of apoptosis correlates with the general state of activation of the lymphoid tissue and not with stage of disease or viral burden. J. Immunol. 1995; 154: 5555 - 5566

33. Katsikis, P. D., Winderlich, E. S., Smith, C.A. and Herzenberg, L. A. Fas antigen stimulation induces marked apoptosis of $T$ lymphocytes in human immunodeficiency virus - infected individuals. J. Exp Med. 1995; 181: $2029-2036$

34. Yu, Y.N., Labbert, B., Screton, G. R., Wolf, D., Kolamus, W. Mongkolsapay, J., Mc Michael, A. J. and Baur, A. S. Induction of Fas ligand expression of HIV involves the interaction of Nef with the T cell receptor E chain. J. Exp. Med. 1999; 189:1489-1496.

35. Westendorp, M. O., Frank, R. Ochsenbamer, C. et al. Sensitization of T cells to CD 95 - mediated apoptosis by HIV-I Tat and gp 120. Nature. 1995; 375:497 - 500.

36. Herbein, G., Mahlknecht, V., Battiwalla, F., Gregersen, P., Pappas, T., Butter, J., O' Brien, W. A. and Verdin E. Apoptosis of CD8 + Tcells is mediated by macrophages through interaction of HIV gp 120 with chemokine receptor CX CR4. Nature, 1998;395: 189 - 194. 